\title{
Diagnostic Performance of the CUT Score in Assessing the Malignancy Risk of Thyroid Nodules
}

\author{
Nese Ersoz GULCELIK ${ }^{1}$, Burcu CANDEMIR ${ }^{1}$, Coskun MERIC ${ }^{1}$, Safak AKIN² ${ }^{2}$ Cem HAYMANA², \\ Ibrahim DEMIRCI ${ }^{2}$, Bagdagul Yuksel GULER ${ }^{1}$, Mithat ESER ${ }^{1}$, Yusuf Alper SONMEZ ${ }^{1}$ \\ ${ }^{1}$ University of Health Sciences, Gulhane Faculty of Medicine, Department of Endocrinology and Metabolism \\ ${ }^{2}$ University of Health Sciences, Gulhane Training and Research Hospital, \\ Department of Endocrinology and Metabolism, Ankara, TURKEY
}

\begin{abstract}
Various scoring systems in order to predict the malignancy risk in thyroid nodules (TNs) have been developed. CUT is one of the novel scoring systems. In this study, we aimed to evaluate its performance in predicting the malignancy risk in TNs and validity in the presence of Hashimoto's thyroiditis (HT). We retrospectively evaluated a total of 252 TNs with a fine needle aspiration biopsy. The CUT scores (Clinical, Ultrasonographic, and Thyroid Cytological scoring system) of the nodules were compared with ATA, ACR-TIRADS and KWAK-TIRADS scores and the histopathology of the nodules. Patients with HT were also compared with m-TIRADS classification. The CUT scores of nodules with malignant histopathology were significantly higher than the benign nodules (3.59 vs. 4.63 , p< 0.001). The area values under the ROC curve of ACR-TIRADS, KWAK-TIRADS, ATA and CUT scoring systems were similar and significantly higher than the reference line [ACR-TIRADS, AUC was 0.762 (95\% Cl: 0.702-0.822, $p<0.001$ ); KWAK-TIRADS, AUC was 0.759 (95\% Cl: 0.699-0.819, p< 0.001); CUT score, AUC was 0.759 (95\% Cl: 0.700-0.819, p<0.001); ATA, AUC was 0.748 (95\% $\mathrm{Cl}$ : 0.687-0.810, $\mathrm{p}<0.001)]$. The areas under the ROC curve were similar when the efficiency of the CUT score was compared with m-TIRADS [CUT score, AUC was 0.772 (95\% Cl: 0.669-0.876, $p<0.001)$; m-TIRADS, AUC was 0.770 (95\% Cl: 0.667-0.874; p< 0.001)] in patients with HT. Our study showed that CUT scoring system was as effective as other scoring systems in predicting the risk of malignancy in thyroid nodules. Furthermore, CUT score is also effective in the presence of HT.
\end{abstract}

Keywords: Thyroid nodule, CUT score, Thyroid cancer

\section{INTRODUCTION}

Thyroid nodules (TNs) are extremely common and are frequently identified in asymptomatic patients as diagnostic imaging methods are widely used. Considering that approximately $95 \%$ of TNs are benign ${ }^{1}$, the primary goal of the management is to avoid unnecessary thyroidectomies. It is well known that even if the presence of a suspicious feature detected in ultrasonography increases the risk of malignancy, further evaluation with fine needle aspiration biopsy (FNAB) is needed. ${ }^{2,3}$ However, careful selection of patients for FNAB and hence surgery is required. Performing FNAB for all TNs may lead to unnecessary interventions and over treatment. Contrary, strict selection criteria can result in a missed diagnosis of a clinically relevant thyroid cancer.

Various standardized systems have developed in order to conduct the risk classification according to the sonographic characteristics of the nodules. KWAK-TIRADS (KWAK-Thyroid Imaging Reporting and Data System) ${ }^{4}$ proposed by Kwak was first established in 2011 followed by the classification recommended in the American Thyroid Association (ATA) guidelines in $2015^{5}$ and the TIRADS classification updated by ACR (American College of Radiology) in 2017 as ACR-TIRADS. ${ }^{6}$ 
A modified TIRADS (m-TIRADS) system was developed by Hang Zhou et al. to classify the risk of malignancy in TNs accompanied by Hashimoto's thyroiditis (HT). ${ }^{7}$ However, these classifications have different specificity and sensitivity values, and problems may occur due to interpretation complexities. ${ }^{89}$ Furthermore, difficulties can still be experienced in selecting patients for surgical treatment, especially in cytologically indeterminate thyroid nodules. Various scoring systems and approaches have been proposed to overcome these problems and to improve the diagnostic efficiency in nodules. ${ }^{10-12}$

Ianni et al. developed a new scoring system on the basis of a meta-analysis of published literature and assigned a matching value to the clinical $(\mathrm{C})$ and ultrasonographic (U) features of TN with increased malignancy risk. The created "CUT" score (Clinical, Ultrasonographic, and Thyroid Cytological scoring system) was derived from " $\mathrm{C}+\mathrm{U}$ " score, along with the five-tiered " $\mathrm{T}$ " representing the cytologic result of the fine-needle aspiration. The "CUT score" evaluates clinical, ultrasonographic, and cytological data to improve the diagnostic efficiency. ${ }^{13}$ In the CUT scoring system, each ultrasonographic risk factor is scored in terms of malignancy risk according to the risk level based on a comprehensive meta-analysis of published studies, contrary to former scoring systems in which a risk classification is made by equally scoring each risk factor. Furthermore, the CUT scoring system also includes clinical features that contribute to the risk of malignancy in the TNs, such as gender, head and/or neck irradiation history, and familial thyroid carcinoma history, and/or familial syndromes associated with a high incidence of thyroid carcinoma. Ianni et al. also integrated cytological results in the CUT scoring system, giving suggestions of management of TNs with different cytological results in order to avoid unnecessary thyroidectomies. ${ }^{13}$

In this study, we aimed to compare the effectiveness of $\mathrm{C}+\mathrm{U}$ score in predicting malignancy risk in TNs with ACR-TIRADS, KWAK-TIRADS and ATA risk classifications. Furthermore, in order to evaluate its effectiveness in a subpopulation of patients with TNs in a thyroiditis background, we aimed to compare $\mathrm{C}+\mathrm{U}$ scores and m-TIRADS scores of this subgroup. The accuracy of managa- ment strategy for indeterminate nodules stated by CUT scoring after integrated cytological information, was also evaluated in thyroid nodules with FNAB results of Bethesda (Bthsd) 1 and 3.

\section{MATERIALS and METHODS}

\section{Patient Selection}

In this study, we retrospectively evaluated patients who had gone for thyroidectomy for benign and malignant TNs in between 2017 and 2020. The study included a total of 252 TNs that were evaluated with FNAB before surgery. One hundred thirty-one of them were benign and 121 of them were malignant according to their histopathological diagnosis. Patients were included in the study that whose clinical history, thyroid ultrasound, laboratory, and cytology results could be obtained.

\section{Methods}

Thyroid ultrasound and FNAB cytology reports were retrospectively evaluated in all patients. $\mathrm{Cy}$ tological diagnosis was made on the basis of the Bethesda (The Bethesda System for Reporting Thyroid Cytopathology) reporting system, which was defined in 2007 and revised in 201714. The Bethesda system uses six categories for thyroid cytology reporting; nondiagnostic (Bthsd1), benign (Bthsd2), atypia of undetermined significance (AUS)/follicular lesion of undetermined significance (FLUS) (Bthsd3), follicular neoplasm/suspicious for follicular neoplasm (SFN) (Bthsd4), suspicious for malignancy (Bthsd5), and malignant (Bthsd6)14. Patients with concomitant thyroidits were determined according to histopathological diagnosis.

\section{Calculation of $\mathrm{C}+\mathrm{U}$ Score}

We calculated the $\mathrm{C}+\mathrm{U}$ components of the CUT score using retrospective clinical (C) and preoperative ultrasonography (U) examination data collection. The following criteria for the clinical component of the $\mathrm{C}+\mathrm{U}$ score were included: gender (male: 0.25 points), history of head and/or neck irradiation ( 0.25 points), family history of thyroid 
carcinoma, and/or familial syndromes associated with a high incidence of thyroid carcinoma $(0.25$ points). For the ultrasonographic component of the $\mathrm{C}+\mathrm{U}$ score, each thyroid nodule was evaluated for the following features: single or multiple nodules (single nodule: 0.25 points), size (maximum value recorded in three dimensions $\geq 4 \mathrm{~cm}: 0.25$ points), shape (anteroposterior diameter taller than wide is considered if greater than the transverse diameter: 1.50 points), margin arrangement (irregular or lobulated: 1.25 points), peripheral halo (incomplete or absent: 1.50 points), echo structure (solid: 1.0 points), echogenicity (hypoechoic: 1.25 points), presence of microcalcifications with or without macrocalcifications (1.25 points), and intranodular vascularization (type 3 diffuse intranodular flow: 0.75 points) 13,15 . $\mathrm{C}+\mathrm{U}$ score risk categories were calculated as described by Ianni et al. ${ }^{13}$

In the study, the recommendation of management of TNs was also evaluated by comparing the CUT scoring system with histopathological outcomes in thyroid nodules with FNAB results of Bthsd 1 and Bthsd 3.

\section{ATA Classification}

According to the ultrasonographic classification of the 2015 ATA guideline, thyroid nodules were included in one of the following risk levels: (1) High risk: solid hypoechoic nodule or partial cystic nodule with solid hypoechoic component together with one or more of the following features; evidence of distorted edge calcification or extra-thyroidal propagation with irregular border (infiltrative, microlobule), microcalcification, taller-than-wide shape, small extrusive soft tissue component; (2) Intermediate risk: Hypoechoic solid nodule without microcalcification, extra-thyroidal invasion or taller-than-wide shape; (3) Low risk: isoechoic or hyperechoic solid nodule or partial cystic nodule without microcalcification, irregular border or extra-thyroidal spread or taller-than-wide shape; (4) Very low risk: partial cystic nodule without spongiform or intermediate or any of the high-risk properties; and (5) Benign: purely cystic nodule without solid component (6).

\section{ACR -TIRADS}

Thyroid nodules that were included in the study were evaluated according to the TIRADS model recommended by ACR. Accordingly, nodules were scored according to their ultrasonographic features and classified in categories ranging from TR1 (benign) to TR5 (highly suspected malignancy). Ultrasound (US) features in ACR-TIRADS: benign (TR1, 0 points), not suspicious (TR2, 2 points), mildly suspicious (TR3, 3 points), moderately suspicious (TR4, 4-6 points), or highly suspicious for malignancy (TR5, 7 points or more). Composition: Cystic or almost completely cystic, 0 points; spongiform, 0 points; mixed cystic and solid, 1 points; solid or almost completely solid, 2 points. Echogenicity: Anechoic, 0 points; hyperechoic or isoechoic, 1 points; hypoechoic, 2 points; very hypoechoic, 3 points. Shape: Taller-than-wide, 3 points. Margin: Smooth, 0 points; properly bounded, 0 points; lobulated or irregular, 2 points; extrathyroidal distribution, 3 points. Echogenic foci: None or large comet-tail artifacts, 0 points; macrocalcification, 1 points; peripheral (rim) calcification, 2 points; punctate echogenic foci, 3 points 6 .

\section{KWAK-TIRADS}

In KWAK-TIRADS, suspicious US features including solid component, hypoechogenicity, marked hypoechogenicity, microlobulated or irregular margins, microcalcifications, and taller-than-wide shape were evaluated. The nodules without any suspicious US features were classified as TIRADS category 3 , and with one suspicious US feature as TIRADS category $4 \mathrm{a}$, with two suspicious US features TIRADS 4b, with three or four suspicious US features TIRADS $4 c$, or with five suspicious US features TIRADS 5.

\section{m-TIRADS}

Among the thyroid nodules included in the study, cases that were histopathologically proved to have accompanied thyroiditis were also evaluated according to the m-TIRADS model. According to the recommendations of Hang Zhou et al. ${ }^{7}$, the total risk score (RS) for each nodule is defined as follows: $\mathrm{RS}=2.1 \mathrm{x}$ (apparent hypoechogenicity $)+1.2 \times($ taller- 


\begin{tabular}{|c|c|c|c|c|c|c|c|}
\hline & $\begin{array}{l}\text { Bethesda } 1 \\
\text { (Malignancy } \\
\text { rate: } 38 \% \text { ) }\end{array}$ & $\begin{array}{l}\text { Bethesda } 2 \\
\text { (Malignancy } \\
\text { rate: } 19 \% \text { ) }\end{array}$ & 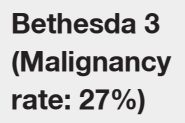 & $\begin{array}{l}\text { Bethesda } 4 \\
\text { (Malignancy } \\
\text { rate: } 33 \% \text { ) }\end{array}$ & $\begin{array}{l}\text { Bethesda } 5 \\
\text { (Malignancy } \\
\text { rate: } 92 \% \text { ) }\end{array}$ & $\begin{array}{l}\text { Bethesda } 6 \\
\text { (Malignancy } \\
\text { rate: } 95 \% \text { ) }\end{array}$ & Total \\
\hline NIFTP & 0 & 0 & 3 & 2 & 0 & 0 & 5 \\
\hline Papillary CA & 8 & 11 & 10 & 5 & 22 & 54 & 110 \\
\hline MicroPTC & 5 & 5 & 9 & 2 & 11 & 19 & $51 / 110$ \\
\hline Follicular CA & 0 & 2 & 0 & 4 & 0 & 0 & 6 \\
\hline Minimally invasive & 0 & 2 & 0 & 3 & 0 & 0 & $5 / 6$ \\
\hline Invasive & 0 & 0 & 0 & 1 & 0 & 0 & $1 / 6$ \\
\hline Total & 8 & 13 & 13 & 11 & 22 & 54 & 121 \\
\hline
\end{tabular}

than wide) $+1.7 x$ (no halo) $+0.6 x$ (irregular border) $+1.2 \times$ (microcalcification or macrocalcification). Next, the m-TIRADS scoring system was divided into 5 categories according to the total RS: m-TIRADS 3 (very low risk, $0 \leq \mathrm{RS}<1.5$ ), $4 \mathrm{a}$ (low risk, $1.5 \leq \mathrm{RS}<3$ ), $4 \mathrm{~b}$ (moderate risk, $3 \leq \mathrm{RS}<4.5$ ), $4 \mathrm{c}$ (high risk, $4.5 \leq \mathrm{RS}<6$ ) and 5 (very high risk, $\mathrm{RS} \geq$ 6)7.

Ethical approval: The study was in accordance with the declaration of Helsinki and approved by Gulhane Teaching and Research Hospital Clinical Research Ethics Committee (Approval no: 2020/ 357).

\section{Statistical Analysis}

All data were analyzed using the statistical package for the social sciences (SPSS 22.0; SPSS Inc., Chicago, Illinois, USA) software. Number, percentage, mean, median, standard deviation (SD), minimum, and maximum values were used for the description of the data analysis. Concordance of continuous variables to normal distribution was measured with the Kolmogorov-Smirnov test. The Mann-Whitney U-test was used for continuous variables and the $x^{2}$-test was used for categorical variables in the comparisons between groups. ROC curve analysis was performed for $\mathrm{C}+\mathrm{U}$ score, ACR-TIRADS, KWAK-TIRADS, m-TIRADS, and ATA classifications. The area under the curve (AUC) and the p-value were calculated. Values of $\mathrm{p}$ less than 0.05 were accepted as statistically significant.

\section{RESULTS}

\section{Patient Characteristics}

Two hundred fifty-two patients (197 females, 55 males) were included in the study. The mean age was $46.2 \pm 13.7$ years. Four patients had a family history of thyroid cancer. One patient had a history of neck irradiation. While total thyroidectomy was performed in $169(67 \%)$ patients, and $83(33 \%)$ patients had hemithyroidectomy. Histopathological examination revealed that $121 / 252(48 \%)$ of patients had thyroid carcinoma and $78(31 \%)$ patients had thyroidits. Forty-three $(35.5 \%)$ of the patients with thyroid cancer and 35 (26.7\%) of the patients with benign pathology had thyroiditis.

\section{Thyroid Nodule Features and Histopathological Diagnosis}

The mean diameter of the nodules was $24 \pm 15 \mathrm{~mm}$. The mean diameter of thyroid nodules with benign pathology was $30.9 \pm 14.9 \mathrm{~mm}$, while the mean diameter of malignant ones was $16.7 \pm 11.2 \mathrm{~mm}(\mathrm{p}<$ 0.001 ). The diamater of the nodule was less than $1 \mathrm{~cm}$ in 38 patients, and the pathological diagnosis was malignant in 33 of them, while benign in 5 nodules. However, tumor size reported by histopathology was less than $1 \mathrm{~cm}$ in 53 (43.8\%) of 121 patients with thyroid carcinoma.

The number of nodules in each category was as follows: Bthsd1, 21/8\%; Bthsd2, 69/27\%; Bthsd3, 48/19\%; Bthsd4, 33/13\%; Bthsd5, 24/10\%; and Bthsd6, 57/22\%. After thyroidectomy, 8 of Bthsd1 


\begin{tabular}{|c|c|c|c|c|c|c|c|}
\hline \multirow[t]{2}{*}{ Cytology } & \multirow[b]{2}{*}{ FNAB, (n) } & \multicolumn{3}{|c|}{ Histopathology } & \multirow[b]{2}{*}{ Benign } & \multirow[b]{2}{*}{ Malign } & \multirow[b]{2}{*}{$p$} \\
\hline & & Surgery, (n) & Benign, n (\%) & Malign, n (\%) & & & \\
\hline Total & 252 & 252 & $131(52)$ & $121(48)$ & 3.59 & 4.63 & $<0.001$ \\
\hline
\end{tabular}

\begin{tabular}{|llll|}
\hline \multicolumn{4}{|l|}{ Table 3. $\mathrm{C}+\mathrm{U}$ risk categories: Malignancy risk regardless of the cytological result } \\
\hline & Benign thyroid nodule, $\mathbf{n}(\%)$ & Malignant thyroid nodule, $\mathbf{n}$ (\%) & Malignancy risk \\
\hline $\mathrm{C}+\mathrm{U}(0-2.5)$ & $18(90)$ & $2(10)$ & Low \\
$\mathrm{C}+\mathrm{U}(2.75-5)$ & $105(57)$ & $80(43)$ & Medium \\
$\mathrm{C}+\mathrm{U}(5.25-10)$ & $4(10)$ & $38(90)$ & High \\
\hline
\end{tabular}

nodules (38\%), 13 of Bthsd2 nodules (19\%), 13 of Bthsd3 nodules (27\%), 11 of Bthsd 4 nodules (33\%), 22 of Bthsd5 nodules (92\%), and 54 (95\%) of Bthsd6 nodules were histopathologically reported as malignant (Table 1). In the nodules reported as malignant, 5 of them (3\%) were NIFTP (noninvasive follicular thyroid neoplasm with papillary-like nuclear features), $110(91 \%)$ of them were papillary carcinomas and $6(5 \%)$ were follicular carcinomas detected through histopathological diagnosis. These malignant nodules and their distribution in cytological categories are shown in Table 1.

\section{“CUT" Score}

The mean $\mathrm{C}+\mathrm{U}$ score of nodules with benign histopathological diagnosis was 3.59 , while the mean $\mathrm{C}+\mathrm{U}$ score of malignant nodules was 4.63. $\mathrm{C}+\mathrm{U}$ score, was found to be significantly higher in malignant cases $(\mathrm{p}<0.001)$ (Table 2). When the $\mathrm{C}+\mathrm{U}$ score was compared in thyroid cancers greater than $1 \mathrm{~cm}$ [papillary thyoid carcinoma (PTC)] and those that were smaller than $1 \mathrm{~cm}$ [papillary thyroid microcarcinoma (mPTC): $53(43.8 \%)$ ], it was found to be similar $(4.67,4.66$, respectively; $\mathrm{p}>0.05)$.

We further evaluated the proposed cut-off values described by Ianni, et al., ${ }^{13}$ and observed that the risk of malignancy in the thyroid nodules was $10 \%$ if the $\mathrm{C}+\mathrm{U}$ value was $\leq 2.5$; it was $43 \%$ if the $\mathrm{C}+\mathrm{U}$ value was between $\geq 2.75$ and $\leq 5$; and finally the risk of malignancy was $90 \%$ if $\mathrm{C}+\mathrm{U}$ value was $\geq$ 5.25 (Table 3).

The management recommendation of the scoring system for TNs included in the study using the scores determined by Ianni, et al., ${ }^{13}$ was also compared with the histopathological results and found out that $34 \%$ and $30 \%$ of nodules in Btsd 1 and $\mathrm{Btsd} 3$ categories that were suggested as "evaluate surgery" came out to be malignant, respectively.

\section{ROC Curve Analysis of the CUT Score, ACR- TIRADS, and ATA Risk Classifications}

\section{Diagnostic performances}

The diagnostic performances of ACR-TIRADS, KWAK-TIRADS, ATA, and CUT scoring systems in terms of predicting malignancy were compared by using ROC curve analysis. According to the ROC curve analysis, the area under the ROC curve of each method was similar, and statistically significant compared to the reference line [ACR-TIRADS, AUC was 0.762 (95\% CI 0.702-0.822, p< $0.001)$; KWAK-TIRADS, AUC was $0.759(95 \%$ CI: 0.699-0.819, p< 0.001); C+U score, AUC was 0.759 (95\% CI: 0.700-0.819, p<0.001); ATA, AUC was 0.748 (95\% CI: 0.687-0.810, $\mathrm{p}<0.001)$ ]. 
International Journal of Hematology and Oncology

Table 4. Comparison of $\mathrm{C}+\mathrm{U}$ score in benign and malignant nodules in patients with and without thyroiditis

\begin{tabular}{|c|c|c|c|c|c|c|}
\hline \multirow[t]{2}{*}{ Thyroiditis } & \multicolumn{2}{|c|}{ Histopathology } & \multicolumn{3}{|c|}{ Mean $\mathrm{C}+\mathrm{U}$ score } & \multirow[b]{2}{*}{$p$} \\
\hline & Benign, n (\%) & Malign, $n(\%)$ & Total & Benign & Malign & \\
\hline Present & $35(45)$ & $43(55)$ & 4.15 & 3.51 & 4.67 & $<0.001$ \\
\hline Not Present & $91(54)$ & 77 (46) & 4.07 & 3.61 & 4.61 & $<0.001$ \\
\hline
\end{tabular}

p: Comparison of thyroid nodules reported as benign and malignant in terms of $\mathrm{C}+\mathrm{U}$ score in histopathology report

\section{Evaluation of Cases with Thyroiditis (m-TI- RADS Risk Classification and CUT Score)}

In patients with thyroiditis proven by histopathological evaluation, the $\mathrm{C}+\mathrm{U}$ score was found to be significantly higher in malignant nodules $(\mathrm{p}<$ 0.001) than the benign ones (Table 4). The CUT score of malignant nodules was found to be similar in patients with and without thyroiditis $(\mathrm{p}>0.05)$. In cases with thyroiditis, the diagnostic performances of m-TIRADS and CUT scores in terms of predicting malignancy were compared using ROC curve analysis, and the area under the ROC curve of each method was found to be similar, and statistically significant compared to the reference line [CUT score, AUC was 0.772 (95\% CI: 0.669 $-0.876, \mathrm{p}<0.001) ; \mathrm{m}$-TIRADS, AUC was 0.770 (95\% CI: 0.667-0.874, p<0.001)].

\section{DISCUSSION}

In this study, CUT score, ACR-TIRADS, KWAKTIRADS, and ATA risk classifications were equally effective in terms of predicting the risk of malignancy. The effectiveness of the CUT score in predicting the risk of malignancy was also evaluated in thyroid nodules accompanied by thyroiditis, and it was found to be similar with the m-TIRADS score.

Various guidelines and scoring systems have been developed to increase the diagnostic accuracy and avoid unnecessary FNAB and thyroidectomy. ${ }^{9}$ The ultrasound-based diagnostic classifications developed for this purpose includes the 2015 ATA guideline classification ${ }^{5}$, KWAK-TIRADS ${ }^{4}$, and ACR-TIRADS $^{6}$ classifications. In addition, in 2016, Hang Zhou, et al. ${ }^{7}$ developed m-TIRADS classification system in thyroid nodules accompa- nied by Hashimoto's thyroiditis. ${ }^{7}$ Several studies have shown that although these classification systems are effective in diagnosis, they have different specificity and sensitivity values. ${ }^{16,17}$ Scoring systems have been developed by some authors to eliminate this complexity and to be more effective in predicting malignancy risk. One of these is the CUT scoring system, which was developed by Ianni, et al. in $2015 .{ }^{13}$ In this scoring system, each ultrasonographic risk factor is scored for malignancy risk according to the risk level based on the previous metanalysis. In addition, in the CUT scoring system, clinical features that contribute to the risk of malignancy in the thyroid nodule are included. ${ }^{13}$ In our study, we showed that the $\mathrm{C}+\mathrm{U}$ score was reliable in distinguishing benign lesions from malignant ones. Moreover, after classifying the score in risk groups as described by Ianni, et al. ${ }^{13}$, the risk of malignancy in a thyroid nodule was $10 \%$ if the $\mathrm{C}+\mathrm{U}$ score was $2.5,43 \%$ if it was between $\geq 2.75$ and $\leq 5$, and was $90 \%$ if it was greater than $\geq 5.25$. These results are found to be similar to the risk rates reported by Ianni, et al. ${ }^{13}$ (9\%, 38\%, and $95 \%$, respectively). ${ }^{13}$ Our findings showed that $\mathrm{C}+\mathrm{U}$ scoring is a valuable tool in predicting malignancy in a population different from the study of Ianni, et al. ${ }^{13}$

Cytological findings were also evaluated in addition to ultrasonographic features in some of these scoring systems. ${ }^{18-20}$ It has been stated that the use of a combined ultrasonographic-cytological index helps the clinician to decide for management of TNs, especially in TNs with an indeterminate cytology. ${ }^{18,19}$ CUT scoring system was evaluated separately in indeterminate thyroid nodules by the same team in 2019 and showed that CUT score could represent a valid aid for the clinician in the management of indeterminate nodules with follicu- 
lar proliferation. ${ }^{15}$ The nodules in Btsd 1 and Btsd3 categories with a CUT score of higher than 2.5 are suggested to be evaluated for surgery by Ianny, et al. ${ }^{14}$ In our study, the recommendation of management of the CUT scoring system for TNs was compared with the histopathological results. The scoring system for TNs with Btsd1 cytology was found to have a detection rate of $34 \%$ for malignant cases. Surgically resected nodules with Btsd1 and Btsd 3 cytology, however, are a selected subset that were repeatedly Btsd1/Btsd3 and/or had worrisome features. Although, the risk of malignancy in Btsd1 nodules is $5-10 \%$, among surgically excised nodules initially reported as Btsd 1 , the malignancy rate is reported as $20 \%(9 \%-32 \%)$. In our study, in the nodules with 2 or more Btsd 1 cytology and with a CUT score of higher than 2.5, the malignancy rate was higher than the reported. Actual risk of malignancy is reported as $14 \%$ in surgically excised nodules with Btsd 3 cytology, (6\%-48\%). ${ }^{5}$ In our study, the risk was $38 \%$ which was higher than the average actual risk of malignancy. So, CUT score which includes the cytology proves to have an additional information in predicting the risk of malignancy in indeterminate nodules.

In a study evaluating the efficacy of classification systems, KWAK-TIRADS and ATA classification showed better diagnostic efficiency than ACR-TIRADS. ${ }^{17}$ However, ACR-TIRADS showed higher specificity. All three classifications (KWAK-TIRADS, ACR-TIRADS and ATA) performed better in distinguishing nodules that are larger than $1 \mathrm{~cm}$. KWAK-TIRADS showed better diagnostic efficiency than other methods in distinguishing nodules $>1 \mathrm{~cm}$ (AUC: 0.92, p< 0.01). ${ }^{17}$ In our study, when the diagnostic efficiency of the CUT score was evaluated by ROC curve analysis, it was found to be as effective as the ACR-TIRADS, KWAKTIRADS and ATA classifications, which were shown to effectively evaluate the malignancy risk between benign and malignant nodules.

Schenke, et al., demonstrated that ACR-TIRADS and KWAK-TIRADS are also reliable in assessing the risk of malignancy in nodules $<1 \mathrm{~cm} \cdot{ }^{21} \mathrm{In}$ our study, CUT score was equally effective with ACR-TIRADS, KWAK-TIRADS, and ATA scoring system in determining malignancy in nodules $<1 \mathrm{~cm}$. The findings of malignancy in this sub- group of nodules may help in the clinical follow-up of which patients should be submitted to an early imaging evaluation or intervention.

Controversial results are reported from various studies examining the relationship between HT and thyroid cancer, in which some authors reported increase in the frequency of thyroid cancer in HT while others failed to show a relationship. ${ }^{22-27}$ In addition, it has been reported that there may be some differences in ultrasonographic features between thyroid nodules with HT and those without HT. ${ }^{28-30}$ In patients with HT, an increase in the incidence of intense calcification and a decrease in the incidence of psammoma bodies have been reported compared to those without HT. ${ }^{28}$ In another study, it was stated that microlobulation or irregular border feature more common in benign nodules in patients with HT. ${ }^{29}$ Additionally, in patients with thyroid cancer and HT, the incidence of irregular border was found to be increased in malign nodules if the gland is hetoregenous. However, in TIRADS classifications, there is no strategy related to these different ultrasonographic features in HT. For this purpose, the m-TIRADS system was developed by Hang Zhou, et al. to classify the risk of malignancy in TNs accompanied by HT. ${ }^{7}$ In this study, prominent hypoechogenicity, taller-than-wide shape, irregular border, microcalcification or macrocalcification and absence of halo were determined as independent risk factors for thyroid cancer in accordance with other studies. ${ }^{7}$ It has been stated that the m-TIRADS classification has high sensitivity and may be useful in making decisions regarding the management of TNs in patients with HT. ${ }^{7}$ In our study, the m-TIRADS scores in patients with HT were significantly higher in malignant nodules. Similarly, the CUT score was useful in distinguishing malignant nodules from benign ones in patients with HT and thyroid nodule. When the diagnostic efficiency of the CUT score and m-TIRADS classification was evaluated with ROC curve analysis, it was found that both methods had similar efficacy. Presence of thyroiditis did not interfere with the diagnostic performance of CUT score in this subgroup of patients.

Our study has several limitations. The retrospective design of the study and the selection of the nodules among the patients who were assigned to 
surgical treatment had resulted in a selection bias; and because of this, a higher rate of thyroid cancer in each Bethesda category was detected in our patients compared to the general population. However, as the Bethesda system has its own pitfalls especially in the indeterminate categories, our results compared the scoring systems of US features with each other by histopathologically proven malignancy and thyroiditis.

In conclusion, our study showed that CUT scoring system was as effective as ACR-TIRADS, KWAKTIRADS and ATA risk classifications in predicting the risk of malignancy in thyroid nodules. Moreover, CUT scoring was similar efficancy with mTIRADS in patients with thyroiditis.

\section{REFERENCES}

1. Mazzaferri EL. Thyroid cancer in thyroid nodules: finding a needle in the haystack. The Am J Med 93: 359-62, 1992.

2. Rago T, Vitti P, Chiovato L, et al. Role of conventional ultrasonography and color flow-doppler sonography in predicting malignancy in 'cold' thyroid nodules. Eur J Endocrinol 138: 41-46, 1998.

3. Papini E, Guglielmi R, Bianchini A, et al. Risk of malignancy in nonpalpable thyroid nodules: predictive value of ultrasound and color-Doppler features. J Clin Endocrinol Metab 87: 1941-1946, 2002.

4. Kwak JY, Han KH, Yoon JH, et al. Thyroid imaging reporting and data system for US features of nodules: a step in establishing better stratification of cancer risk. Radiology 260 : 892-899, 2011.

5. Haugen BR, Alexander EK, Bible KC, et al. 2015 American Thyroid Association Management Guidelines for Adult Patients with Thyroid Nodules and Differentiated Thyroid Cancer: The American Thyroid Association Guidelines Task Force on Thyroid Nodules and Differentiated Thyroid Cancer. Thyroid 26: 1-133, 2016.

6. Tessler FN, Middleton WD, Grant EG, et al. ACR Thyroid Imaging, Reporting and Data System (TI-RADS): White Paper of the ACR TI-RADS Committee. J Am Coll Radiol 14: 587-595, 2017.

7. Zhou H, Yue WW, Du LY, et al. A Modified Thyroid Imaging Reporting and Data System (mTI-RADS) For Thyroid Nodules in Coexisting Hashimoto's Thyroiditis. Sci Rep 6: 26410, 2016.

8. Horvath E, Majlis S, Rossi R, et al. An ultrasonogram reporting system for thyroid nodules stratifying cancer risk for clinical management. J Clin Endocrinol Metab 94: 1748-1751, 2009.
9. Park JY, Lee HJ, Jang HW, et al. A proposal for a thyroid imaging reporting and data system for ultrasound features of thyroid carcinoma. Thyroid 19: 1257-1264, 2009.

10. Garino F, Deandrea M, Motta M, et al. Diagnostic performance of elastography in cytologically indeterminate thyroid nodules. Endocrine 49: 175-183, 2015.

11. Trimboli P, Treglia G, Sadeghi R, et al. Reliability of real-time elastography to diagnose thyroid nodules previously read at FNAC as indeterminate: a meta-analysis. Endocrine 50: 335343, 2015.

12. Nasrollah N, Trimboli P, Guidobaldi L, et al. Thin core biopsy should help to discriminate thyroid nodules cytologically classified as indeterminate. A new sampling technique. Endocrine 43: 659-665, 2013.

13. lanni $\mathrm{F}$, Campanella $\mathrm{P}$, Rota CA, et al. A meta-analysis-derived proposal for a clinical, ultrasonographic, and cytological scoring system to evaluate thyroid nodules: the "CUT" score. Endocrine 52: 313-321, 2016.

14. Ianni F, Pascucci D, Paragliola RM, et al. Follow-Up or Surgery for Indeterminate Thyroid Nodules: Could the CUT Score Application Be a Support for Decision-Making in the Preoperative Assessment? Thyroid 30: 65-71, 2020.

15. Cibas ES, Ali SZ. The 2017 Bethesda System for Reporting Thyroid Cytopathology. Thyroid 27: 1341-1346, 2017.

16. Wang D, Du LY, Sun JW, et al. Evaluation of thyroid nodules with coexistent Hashimoto's thyroiditis according to various ultrasound-based risk stratification systems: A retrospective research. Eur J Radiol 131: 109059, 2020.

17. Gao L, Xi X, Jiang $Y$, et al. Comparison among TIRADS (ACR TI-RADS and KWAK- TI-RADS) and 2015 ATA Guidelines in the diagnostic efficiency of thyroid nodules. Endocrine 64: 90-96, 2019.

18. Tomimori EK, Camargo RY, Bisi $\mathrm{H}$, et al. Combined ultrasonographic and cytological studies in the diagnosis of thyroid nodules. Biochimie 81: 447-452, 1999.

19. Camargo RY, Tomimori EK, Knobel M, et al. Preoperative assessment of thyroid nodules: role of ultrasonography and fine needle aspiration biopsy followed by cytology. Clinics (Sao Paulo, Brazil) 62: 411-418, 2007.

20. Hong MJ, Na DG, Baek JH, et al. Cytology-Ultrasonography Risk-Stratification Scoring System Based on Fine-Needle Aspiration Cytology and the Korean-Thyroid Imaging Reporting and Data System. Thyroid 27: 953-959, 2017.

21. Schenke S, Klett R, Seifert P, et al. Diagnostic Performance of Different Thyroid Imaging Reporting and Data Systems (Kwak-TIRADS, EU-TIRADS and ACR TI-RADS) for Risk Stratification of Small Thyroid Nodules ( $\leq 10 \mathrm{~mm}$ ). J Clin Med 9: 236, 2020.

22. Mukasa K, Noh JY, Kunii Y, et al. Prevalence of malignant tumors and adenomatous lesions detected by ultrasonographic screening in patients with autoimmune thyroid diseases. Thyroid 21: 37-41, 2011. 
23. Cunha LL, Ferreira RC, Marcello MA, et al. Clinical and pathological implications of concurrent autoimmune thyroid disorders and papillary thyroid cancer. J Thyroid Res 2011: 387062, 2011.

24. Azizi G, Malchoff CD. Autoimmune thyroid disease: a risk factor for thyroid cancer. Endocrine Practice 17: 201-209, 2011.

25. Kim KW, Park YJ, Kim EH, et al. Elevated risk of papillary thyroid cancer in Korean patients with Hashimoto's thyroiditis. Head Neck 33: 691-695, 2011.

26. Zhang $\mathrm{L}$, $\mathrm{Li} \mathrm{H}$, Ji QH, et al. The clinical features of papillary thyroid cancer in Hashimoto's thyroiditis patients from an area with a high prevalence of Hashimoto's disease. BMC Cancer 12: 610, 2012.

27. Gul K, Dirikoc A, Kiyak G, et al. The association between thyroid carcinoma and Hashimoto's thyroiditis: the ultrasonographic and histopathologic characteristics of malignant nodules. Thyroid 20: 873-878, 2010.

28. Ohmori N, Miyakawa M, Ohmori K, et al. Ultrasonographic findings of papillary thyroid carcinoma with Hashimoto's thyroiditis. Intern Med (Tokyo) 46: 547-550, 2007.

29. Park M, Park SH, Kim EK, et al. Heterogeneous echogenicity of the underlying thyroid parenchyma: how does this affect the analysis of a thyroid nodule? BMC Cancer 13: 550, 2013.

30. Durfee SM, Benson CB, Arthaud DM, et al. Sonographic appearance of thyroid cancer in patients with Hashimoto thyroiditis. J Ultrasound Med 34: 697-6704, 2015.

\section{Correspondence:}

\section{Dr. Safak AKIN}

Saglik Bilimleri Universitesi, Gulhane Tip Fakultesi

Endokrinoloji ve Metabolizma Anabilim Dali

Etlik, ANKARA / TURKEY

Tel: (+90-532) 2206940

e-mail: safakcavus@gmail.com

\section{ORCIDs:}

$\begin{array}{ll}\text { Nese Ersoz Gulcelik } & 0000-0001-8212-5752 \\ \text { Burcu Candemir } & 0000-0002-5687-0756 \\ \text { Coskun Meric } & 0000-0002-0766-0517 \\ \text { Safak Akin } & 0000-0003-3886-0500 \\ \text { Cem Haymana } & 0000-0002-6866-2364 \\ \text { Ibrahim Demirci } & 0000-0002-6808-8550 \\ \text { Bagdagul Yuksel Guler } & 0000-0002-8023-2666) \\ \text { Mithat Eser } & 0000-0002-1430-1723 \\ \text { Yusuf Alper Sonmez } & 0000-0002-9309-7715\end{array}$

